\title{
Intestinal CFTR expression alleviates meconium ileus in cystic fibrosis pigs
}

\author{
David A. Stoltz,1,2 Tatiana Rokhlina, ${ }^{1}$ Sarah E. Ernst,, ${ }^{1,3}$ Alejandro A. Pezzulo, ${ }^{1}$ \\ Lynda S. Ostedgaard, ${ }^{1}$ Philip H. Karp, ${ }^{1,3}$ Melissa S. Samuel, ${ }^{4}$ Leah R. Reznikov, ${ }^{1}$ Michael V. Rector, ${ }^{1}$ \\ Nicholas D. Gansemer, ${ }^{1}$ Drake C. Bouzek, ${ }^{1}$ Mahmoud H. Abou Alaiwa, ${ }^{1}$ Mark J. Hoegger, ${ }^{1}$ \\ Paula S. Ludwig, ${ }^{1}$ Peter J. Taft, ${ }^{1}$ Tanner J. Wallen, ${ }^{5}$ Christine Wohlford-Lenane, ${ }^{6}$ \\ James D. McMenimen, ${ }^{1}$ Jeng-Haur Chen,, ${ }^{1,3}$ Katrina L. Bogan, ${ }^{1}$ Ryan J. Adam, ${ }^{1}$ Emma E. Hornick, ${ }^{1}$ \\ George A. Nelson IV, ${ }^{1}$ Eric A. Hoffman, ${ }^{7}$ Eugene H. Chang, ${ }^{5}$ Joseph Zabner, ${ }^{1}$ Paul B. McCray Jr., ${ }^{6}$
}

Randall S. Prather, ${ }^{4}$ David K. Meyerholz, ${ }^{8}$ and Michael J. Welsh ${ }^{1,3}$

\begin{abstract}
${ }^{1}$ Department of Internal Medicine, ${ }^{2}$ Department of Biomedical Engineering, and ${ }^{3}$ Howard Hughes Medical Institute,
University of lowa Roy J. and Lucille A. Carver College of Medicine, lowa City, lowa, USA. ${ }^{4}$ Division of Animal Science, University of Missouri,

Columbia, Missouri, USA. ${ }^{5}$ Department of Otolaryngology, Head and Neck Surgery, ${ }^{6}$ Department of Pediatrics, ${ }^{7}$ Department of Radiology, and

${ }^{8}$ Department of Pathology, University of lowa Roy J. and Lucille A. Carver College of Medicine, lowa City, lowa, USA.
\end{abstract}

\begin{abstract}
Cystic fibrosis (CF) pigs develop disease with features remarkably similar to those in people with CF, including exocrine pancreatic destruction, focal biliary cirrhosis, micro-gallbladder, vas deferens loss, airway disease, and meconium ileus. Whereas meconium ileus occurs in $15 \%$ of babies with CF, the penetrance is $100 \%$ in newborn CF pigs. We hypothesized that transgenic expression of porcine $C F$ transmembrane conductance regulator $(p C F T R) \mathrm{cDNA}$ under control of the intestinal fatty acid-binding protein $(i F A B P)$ promoter would alleviate the meconium ileus. We produced $5 \mathrm{CFTR}^{-/-}$; $T$ gFABP>pCFTR lines. In 3 lines, intestinal expression of CFTR at least partially restored CFTR-mediated anion transport and improved the intestinal phenotype. In contrast, these pigs still had pancreatic destruction, liver disease, and reduced weight gain, and within weeks of birth, they developed sinus and lung disease, the severity of which varied over time. These data indicate that expressing CFTR in intestine without pancreatic or hepatic correction is sufficient to rescue meconium ileus. Comparing CFTR expression in different lines revealed that approximately $20 \%$ of wild-type CFTR mRNA largely prevented meconium ileus. This model may be of value for understanding CF pathophysiology and testing new preventions and therapies.
\end{abstract}

\section{Introduction}

Cystic fibrosis (CF) is caused by mutations in the gene encoding the CF transmembrane conductance regulator (CFTR). Loss of CFTR function causes pancreatic insufficiency, focal biliary cirrhosis, infertility, and recurrent and chronic airway infections that destroy the lung (1-3). In addition, up to $20 \%$ of babies with CF are born with meconium ileus $(4,5)$. Intestinal obstruction most commonly occurs in the distal ileum to proximal colon and often requires surgical intervention to relieve the obstruction $(6,7)$. The pathogenesis of meconium ileus remains uncertain, but may result from loss of CFTR-mediated $\mathrm{Cl}^{-}$and/or $\mathrm{HCO}_{3}{ }^{-}$transport by the intestinal epithelium and/or from pancreatic dysfunction (8-12).

To better understand the pathogenesis of $\mathrm{CF}$, we developed a porcine model of CF (13-15) and found that $C F T R^{-/-}$and CFTR ${ }^{\triangle F 508 / \triangle F 508}$ pigs (hereafter called CF pigs) display many of the same features as humans with $\mathrm{CF}$, including meconium ileus, exocrine pancreatic destruction, focal biliary cirrhosis, micro-gallbladder, vas deferens abnormalities, and airway disease (1,14-17). However, in contrast with humans, the penetrance of meconium ileus is $100 \%$ in newborn $\mathrm{CF}$ pigs. Features of meconium ileus in CF pigs closely replicate those observed in humans with $\mathrm{CF}$, including obstruction by meconium in the distal small intestine, atretic intestinal segments, and microcolon (14-16). The meconium ileus in CF pigs would be lethal

Conflict of interest: David A. Stoltz, Michael J. Welsh, and the University of Iowa Foundation have applied for a patent related to the work reported in this paper. Michael J. Welsh is a cofounder of Exemplar Genetics.

Citation for this article: J Clin Invest. 2013;123(6):2685-2693. doi:10.1172/JCI68867. if not corrected soon after birth. In some cases, Gastrografin enemas can relieve meconium ileus obstruction and result in avoidance of surgical correction in newborn babies with CF $(18,19)$. However, attempts to nonsurgically correct the intestinal obstruction in CF pigs were unsuccessful, in part due to the atretic intestinal segments. Surgical placement of an ileostomy bypassed the obstruction, allowing CF pigs to survive $(14,15,20)$. Despite the ability to surgically treat meconium ileus in CF pigs, the procedure can be associated with morbidity and mortality. Moreover, surgical correction is not feasible in some CF pigs due to complications associated with meconium ileus, including intestinal atresia and in utero intestinal perforation. That, plus the associated costs, precludes surgical correction as a means to obtain older CF pigs on a routine basis.

In an attempt to alleviate the meconium ileus phenotype in $\mathrm{CF}$ pigs, we were inspired by the approach of Zhou and colleagues (21). They used the rat intestinal fatty acid-binding protein $(i F A B P)$ promoter $(22,23)$, which is primarily expressed in the intestine (23), to express human CFTR in CF mice. Here, we adopted that strategy and we asked three questions. First, would expressing CFTR under control of the $i F A B P$ promoter prevent meconium ileus in CF pigs? If we observed correction, then these findings would help us better understand the pathogenesis of meconium ileus. Second, knowing that different $i F A B P$-CFTR transgenic cell lines would likely have differing levels of CFTR expression, we asked how much CFTR function is required to prevent meconium ileus in $\mathrm{CFTR}^{-/-}$pigs. Finally, if this strategy alleviated the meconium ileus, would these animals develop airway disease similar to that observed in humans with $\mathrm{CF}$ ? 
A

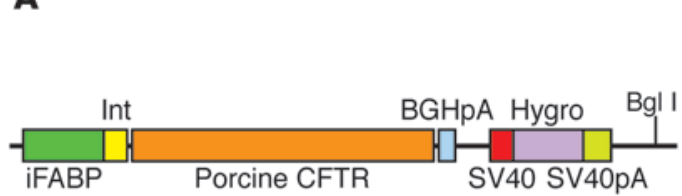

C
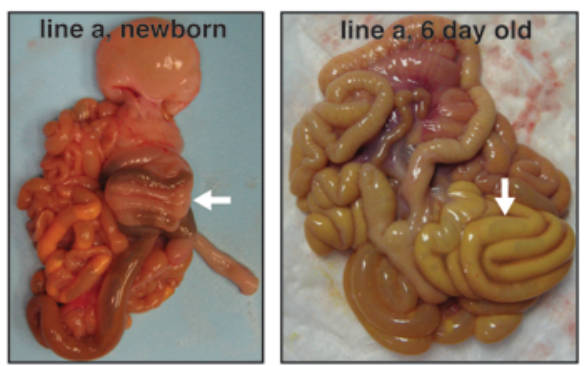

B
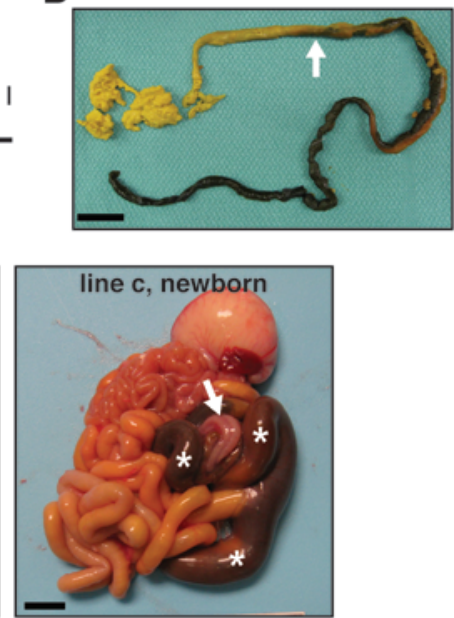

\section{Figure 1}

Intestinal FABP promoter-driven CFTR expression alleviates meconium ileus in CF pigs. (A) Schematic of the transgene vector with porcine CFTR cDNA driven by the rat iFABP promoter (nt -1788 to +28 ), flanked by the intervening sequence and the bovine growth hormone poly-A (BGHpA) and followed by a hygromycin cDNA sequence flanked by SV4O and SV4O poly-A signal sequences. $B g / l$ restriction site is denoted. (B) Gross image of a meconium plug (green-black colored portion of stool) that was passed by a CFTR ${ }^{-1} ; \operatorname{TgFABP}>p C F T R$ pig (line e) following an enema at approximately 18 hours after birth. A transition to normal-appearing stool (yellow-green stool denoted by arrow) was observed after meconium passage. Scale bar: $2 \mathrm{~cm}$. (C) Images of the gastrointestinal tracts of CFTR ${ }^{-1-} ; T g F A B P>p C F T R$ piglets. At birth, in 3 of the 5 CFTR ${ }^{-1-}$; TgFABP $>$ pCFTR lines, meconium ileus was improved. Meconium ileus lesions ranged from improved (left panel, line a) with normal-sized intestine and spiral colon (arrow) to severe meconium obstruction (right panel, line c, white asterisks) often located in the spiral colon with distal microcolon (arrow). Middle panel: gross image from the intestinal tract of a CFTR ${ }^{--} ;$TgFABP $>$pCFTR pig (line a) at 6 days of age. The intestine and spiral colon (arrow) are of normal size and coloration. Scale bar: $1.3 \mathrm{~cm}$.

\section{Results}

Generation of $\mathrm{CFTR}^{-/-}$; $T \mathrm{TFABP}>\mathrm{pCFTR}$ pigs. We hypothesized that intestinal expression of CFTR in $C F T R^{-1-}$ pigs would rescue the meconium ileus phenotype or produce a milder phenotype amenable to correction with nonsurgical techniques. To express CFTR in the intestinal tract, we used wild-type porcine CFTR ( $P C F T R$ ) driven by the rat $i F A B P$ gene promoter $(21,22)$. To produce $C F T R^{-1}$ gut-corrected pigs (hereafter called $C F T R^{-1-} ; T g F A B P>p C F T R$ pigs), $C F T R^{-1-}$ fetal fibroblasts (male) were transfected with a rat $i F A B P$ PCFTR construct (Figure 1A). Antibiotic selection was performed followed by confirmation of positive clones with Southern blotting. Positive clones were expanded and used as nuclear donors for transfer to enucleated oocytes. Somatic cell nuclear transfer embryos were transferred to surrogate females, and piglets were delivered at term. Five different clones (a-e) were transferred individually to surrogate females.

The iFABP-pCFTR transgene rescues the meconium ileus phenotype in $\mathrm{CFTR}^{-1-}$ pigs. Not knowing whether this transgenic approach would work, we decided to enhance the likelihood of correcting meconium ileus by treating the majority of animals with Gastrografin enemas after birth. This strategy is used to treat meconium ileus in human newborns with CF $(18,19)$, but failed to relieve the intestinal obstruction in CF pigs. The response to the enema was variable among litters. Following the initial enema, we recovered either nothing, meconium pellets, or small white mucous plugs $(\sim 2 \mathrm{~mm}$ in diameter). To enhance removal of meconium from the intestinal tract, repeat Gastrografin enemas were performed at 8 - to 12 -hour intervals until either all of the meconium was evacuated (Figure 1B) or the animals failed to pass meconium despite repeat enemas. Animals that had no or minimal meconium output despite good oral intake were euthanized. Pigs that passed meconium were transitioned to a piglet milk diet.

Here we report data on 15 piglets from 5 transgenic lines. Piglets from lines a, d, and e had an improved intestinal phenotype. Figure 1C shows images of $\mathrm{CFTR}^{-/-} ; \mathrm{TgFABP}>\mathrm{pCFTR}$ intestine from newborn and 6-day-old pigs (line a). For comparison, Figure 1C (line c) shows intestine from a newborn pig that had severe meconium ileus, like that in newborn CFTR ${ }^{-/-}$pigs $(14,16)$. Pigs from lines a, $d$, and e had less severe small intestine obstruction, intestinal atresia, and microcolon as well as greater meconium passage. In contrast, piglets from lines $\mathrm{b}$ and $\mathrm{c}$ did not differ from $\mathrm{CFTR}^{-/-}$ pigs (Figure 2A). These findings indicate that expressing $P C F T R$ under control of the rat $i F A B P$ promoter can rescue the meconium ileus phenotype.

CFTR is expressed and functional in the ileum of pigs with a rescued intestinal phenotype. These data suggested that differing levels of CFTR expression might be responsible for the variable phenotypes. To test this hypothesis, we used quantitative RT-PCR to measure CFTR mRNA in ileal segments of newborn pigs. CFTR transcripts varied in the different lines, with lines a and e showing substantial CFTR mRNA, lines b and c showing levels not different from that of $\mathrm{CFTR}^{-/-}$ileum, and line $\mathrm{d}$ showing intermediate values (Figure 2B).

We used immunocytochemistry to localize CFTR in ileum. CFTR was undetectable in $\mathrm{CFTR}^{-1-}$ tissue (Figure 2C). In wild-type ileum, CFTR localized to the apical membrane of crypt, but not villous epithelia, consistent with other reports of endogenous CFTR localization in the intestine $(14,24-26)$. In line e, we detected CFTR variably present at the apical membrane of crypt epithelial cells. Transgenederived CFTR expression in crypt cells is consistent with the localization of CFTR in wild-type pigs and with the expression pattern of intestinal FABP in human intestine $(14,27)$. Interestingly, in mice transgenic for the rat $i F A B P$ promoter driving $b C F T R$ expression, CFTR was localized in both the villous (21) and crypt epithelia (28).

To learn whether the recombinant PCFTR was functional, we excised ileal segments, mounted them in Ussing chambers, and assayed transepithelial currents. We added forskolin and 3-isobutyl-2-methylxanthine (IBMX) to increase cellular concentrations of cAMP and phosphorylate and activate CFTR. Forskolin and IBMX increased short-circuit current (Isc) in ileal segments of $\mathrm{CFTR}^{+/+}$, but not typically in $\mathrm{CFTR}^{-/-}$intestine (Figure 2, D and E). The response varied in ileum from the different transgenic animals (Figure $2 \mathrm{D}$ ). Lines a and e had $\Delta \mathrm{Isc}_{\mathrm{F} \& \mathrm{I}}$ responses that were approximately $60 \%$ or greater than that in wild-type ileum. Ileum from lines $\mathrm{b}$ and $\mathrm{c}$ lacked significant $\Delta \mathrm{Isc}_{\mathrm{F} \& \mathrm{I}}$ and was similar to $C F T R^{-/-}$ tissue. Line $\mathrm{d}$ had an intermediate $\Delta \mathrm{Isc}_{\mathrm{F} \& \mathrm{I}}$ response. 
A

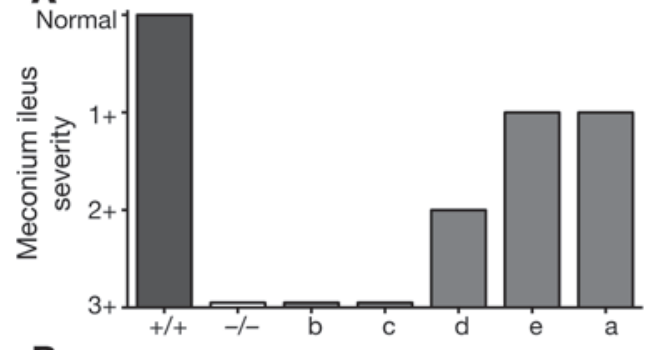

B

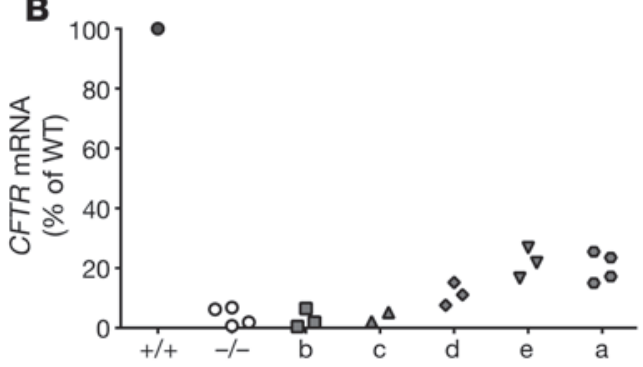

D
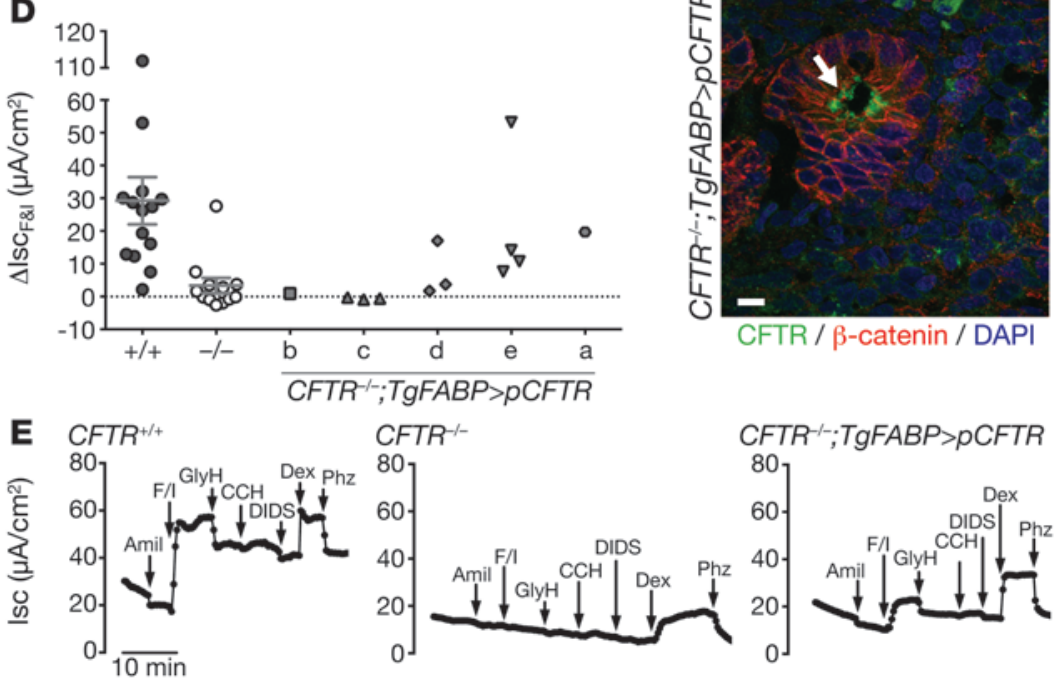

C
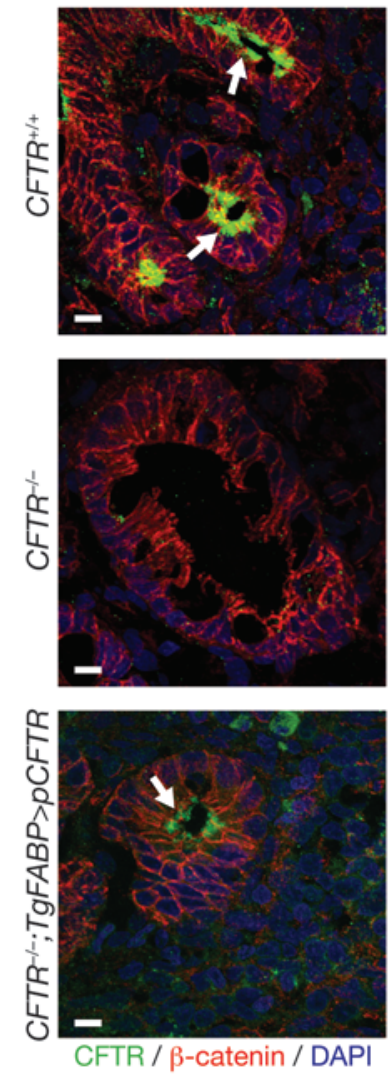

10 $10 \mu \mathrm{M}$ forskolin/100 $\mu \mathrm{M}$ IBMX (F/I), apical $100 \mu \mathrm{M}$ $\mathrm{GlyH}$, basolateral $100 \mu \mathrm{M}$ carbachol $(\mathrm{CCH})$, apical $100 \mu \mathrm{M}$ DIDS, apical $5 \mathrm{mM}$ dextrose (Dex), and apical $200 \mu \mathrm{M}$ phlorizin (Phz).
CFTR transcripts and transepithelial current correlate with rescue of meconium ileus. We compared mRNA levels, secretory current, and meconium ileus to assess the relationship between the amount of CFTR and the phenotype. As the abundance of CFTR transcripts increased, secretory current increased (Figure 3A). There was a similar relationship between CFTR mRNA and the meconium ileus phenotype (Figure $3 \mathrm{~B}$ ). These data suggest that approximately $20 \%$ of wild-type CFTR mRNA and approximately $60 \%$ of $\mathrm{Cl}^{-}$secretory current was sufficient to rescue the meconium ileus phenotype. Whereas approximately $10 \%$ of CFTR mRNA and $25 \%$ of wild-type CFTR-mediated anion secretion was not sufficient to prevent meconium ileus.

\section{Figure 3}

CFTR expression and secretory current correlate with meconium ileus phenotype. (A) lleal $\triangle \mathrm{ISC}_{\mathrm{F} \& \mathrm{I}}$ versus ileal CFTR mRNA levels in the different CFTR $^{-1-}$; TgFABP $>$ pCFTR lines. (B) Meconium ileus severity versus ileal CFTR mRNA levels in the different transgenic lines. Data are shown as a percentage of wild-type levels $\left(\right.$ CFTR $\left.^{+/+}[+/+]\right) .-/-$, CFTR $^{-/-}$ animals. Individual labels denote the different cell lines.

CFTR $^{-1-} ; \mathrm{TgFABP}>\mathrm{pCFTR}$ pigs have CF-related pancreas, gallbladder, and liver disease. In all of these transgenic lines, we observed a similar degree of pancreatic destruction (Supplemental Figure 1, A and B; supplemental material available online with this article; doi:10.1172/JCI68867DS1), micro-gallbladder (Supplemental Figure 1, C and D), and focal biliary cirrhosis (Supplemental Figure 1E) as previously reported in newborn $C F T R^{-/-}$and $C F T R^{\triangle F 508 / \triangle F 508}$ pigs $(14-16,20)$. These findings indicate that the

A

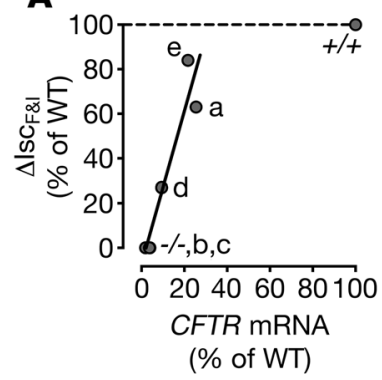

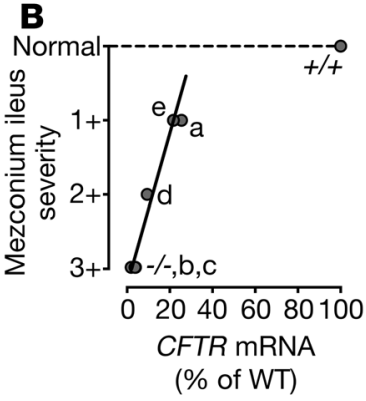


A
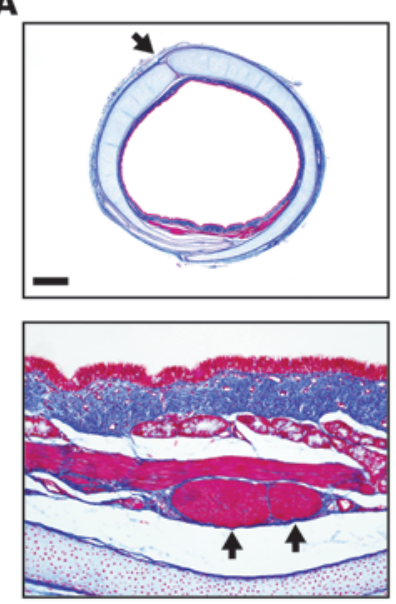

B
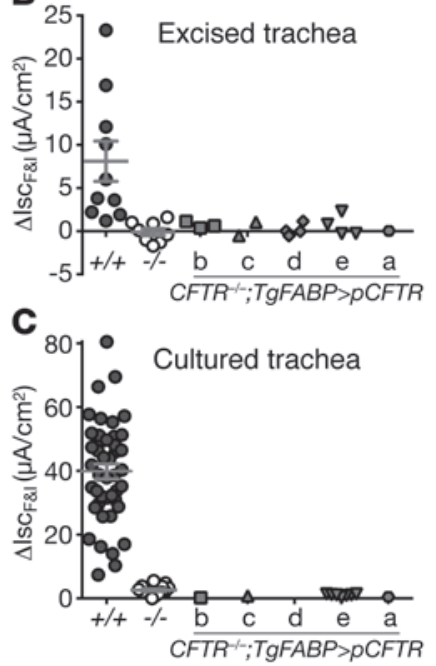

iFABP-pCFTR transgene did not correct other digestive system manifestations of CF.

CFTR is not present in the airways of $C F T R^{-1}$;TgFABP>pCFTR pigs. In rodents, expression of the endogenous $i F A B P$ gene is most abundant in the intestinal tract (23). However, when human CFTR was expressed under control of the rat $i F A B P$ promoter in CFTR-null mice, some lines exhibited extra-intestinal human CFTR expression, including in the lungs (21). On gross examination, tracheas from all 5 CFTR ${ }^{-/}$; $T g F A B P>$ PCFTR lines had narrowed airway lumens, cartilaginous ring defects, and abnormal-appearing airway smooth muscle bundles, as we previously observed in neonatal $\mathrm{CF}$ pigs and humans with $\mathrm{CF}$ (Figure $4 \mathrm{~A}$ and ref. 29).

To further test for CFTR function, we harvested fresh tracheal tissue from newborn pigs, mounted the excised tissue in Ussing chambers, and measured transepithelial currents. We added amiloride to block epithelial $\mathrm{Na}^{+}$channels and 4,4-diisothiocyanostilbene-2,2-disulfonic acid (DIDS) to block non-CFTR $\mathrm{Cl}^{-}$channels. Subsequent addition of forskolin and IBMX increased Isc in $\mathrm{CFTR}^{+/}$tracheas, but failed to substantially increase Isc in $\mathrm{CFTR}^{--}$or any of the tracheas from the CFTR - ; $T g F A B P>p C F T R$ lines (Figure 4B and Supplemental Figure 2B). We obtained qualitatively similar results with cultured tracheal epithelia (Figure 4C) and nasal turbinate epithelium (Supplemental Figure 2C), including the lack of cAMP-stimulated transepithelial $\mathrm{Cl}^{-}$

\section{Figure 5}

CFTR $R^{--}$;TgFABP $>$pCFTR pigs develop airway disease postnatally. (A) Both CFTR ${ }^{-1-}$ (white circles: $n=5$ ) and CFTR ${ }^{-1-}$;gFABP $>$ pCFTR (gray circles: $n=6$ ) pigs gained less weight than did non-CF pigs (black circles: $\mathrm{CFTR}^{+++}, n=5 ; \mathrm{CFTR}^{+/-}, n=1$ ). Data are mean $\pm \mathrm{SEM} .{ }^{*} P<0.01$ for both $C F$ and gut-corrected CF pigs compared with non-CF pigs. (B) Microscopic image of the pancreas from case no. 3. Pancreas was composed mostly of adipose tissue (asterisks) with sparse remnant pancreatic tissue and intact islets (arrows). (C) Sinus x-ray CT image from a CFTR ${ }^{-1-}$;gFABP $>$ pCFTR pig (case no. 4) at approximately 3 weeks of age. Bilateral ethmoid sinus opacification (arrows) was present on the sinus CT scan. (D) Case no. 4, severe sinus disease with mucopurulent material obstructing the sinus lumen with submucosal (Bowman's) gland hypertrophy. dPAS stain. (E) Case no. 4, mucopurulent debris obstructing a small bronchiole. H\&E stain. (F) Case no. 4, bronchus with goblet cell hyperplasia. dPAS stain. Scale bars: $167 \mu \mathrm{m}$ (B); $90 \mu \mathrm{m}(\mathbf{D}) ; 87 \mu \mathrm{m}(\mathbf{E}) ; 29 \mu \mathrm{m}$ (F).

\section{Figure 4}

CFTR $^{-1-}$;TgFABP $>$ pCFTR pigs lack CFTR-mediated electrolyte transport in the airways. (A) Microscopic images of trachea from a CFTR ${ }^{-1-}$; $T g F A B P>p C F T R$ newborn pig. The tracheas were irregularly shaped, had cartilage ring defects in the anterior trachea (top panel, arrow), and areas of abnormal-appearing airway smooth muscle bundles (bottom panel, arrows) (30). Scale bars: $820 \mu \mathrm{m}$ (top panel); $82 \mu \mathrm{m}$ (bottom panel). (B) Changes in short-circuit current (Isc) following addition of forskolin/IBMX $\left(\triangle \mathrm{ISC}_{\mathrm{F} \& \mathrm{I}}\right)$ to freshly excised tracheal segments from newborn $\mathrm{CFTR}^{+/+}, \mathrm{CFTR}^{-/-}$, and $\mathrm{CFTR}^{-/-;}$TgFABP $>$pCFTR piglets. (C) $\triangle \mathrm{ISC}_{\mathrm{F} \& \mathrm{l}}$ in cultured tracheal epithelia from newborn $\mathrm{CFTR}^{+/+}, \mathrm{CFTR}^{-1-}$, and CFTR $^{-/-} ;$TgFABP $>$PCFTR piglets (no sample was available for line d). Prior to forskolin/IBMX treatment, tissues were sequentially exposed to amiloride and DIDS. Symbols represent individual animals. Line represents mean \pm SEM. Data for lines $b, d$, and $e$ in $\mathbf{B}$ and line e in $\mathbf{C}$ did not differ significantly from data for $\mathrm{CFTR}^{-1}$.

current. Moreover, these data are quantitatively similar to those we previously reported for upper and lower airways in $\mathrm{CF}$ and non-CF pigs (30). These data indicated that the iFABP-pCFTR transgene generates no appreciable CFTR expression in the proximal airways and suggested that the transgenic pigs might develop airway disease.

CFTR ${ }^{-/}$;TgFABP>PCFTR pigs survive without surgical correction of

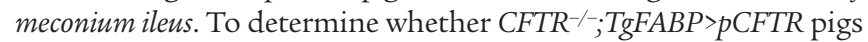
would develop lung disease, we followed 6 transgenic $\mathrm{CF}$ pigs for 3 or more weeks (Supplemental Table 1). Case nos. 1-3 were treated with Gastrografin enemas at birth and case nos. 4-6 survived without an enema. Similar to $\mathrm{CFTR}^{-/}$pigs and gut-corrected CF mice $(31,32)$, CFTR $^{-1}$; $T$ TgFABP>PCFTR pigs had reduced growth (Figure $5 \mathrm{~A}$ ). These data suggest that the growth defect in CF pigs is not dependent upon intestinal CFTR expression (32). We euthanized pigs when they developed signs of respiratory disease or complications of gastric ulcer or at planned time points.

A

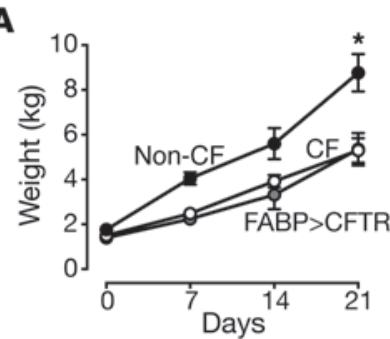

B
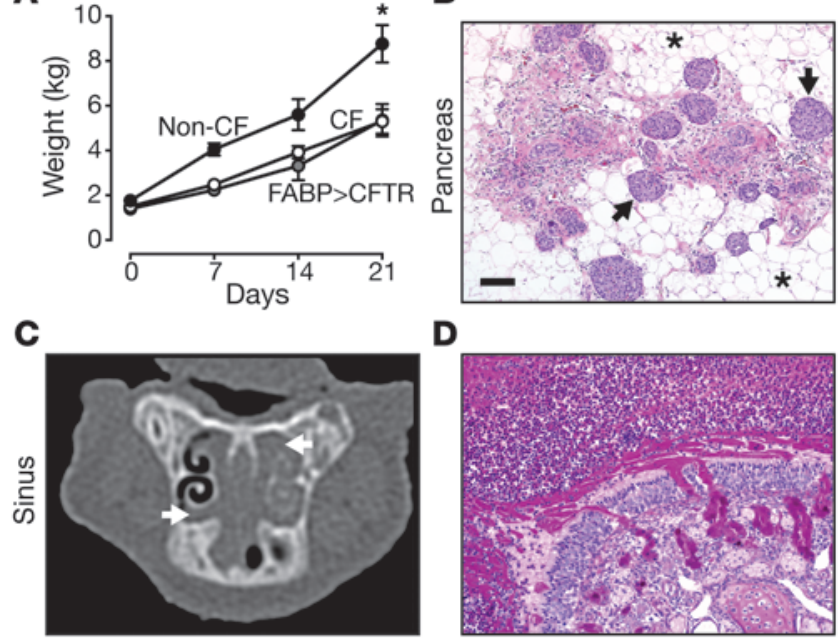

D

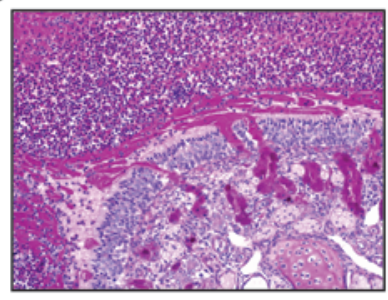

E

F

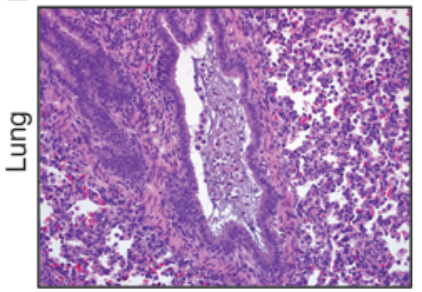

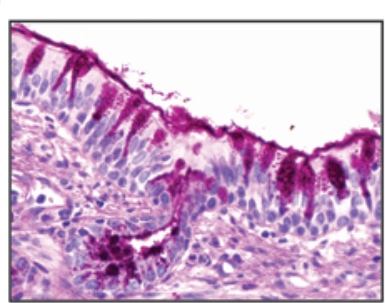


A
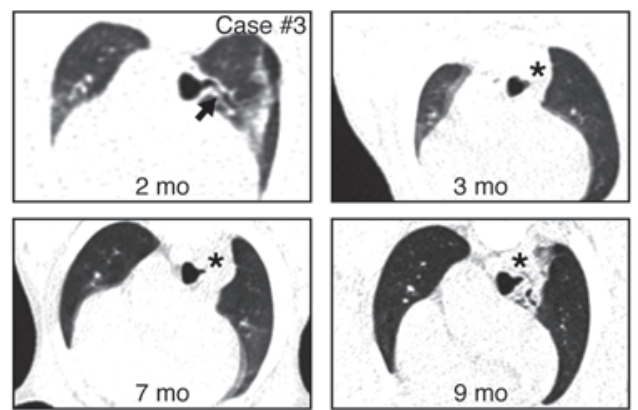

B

Case \#3
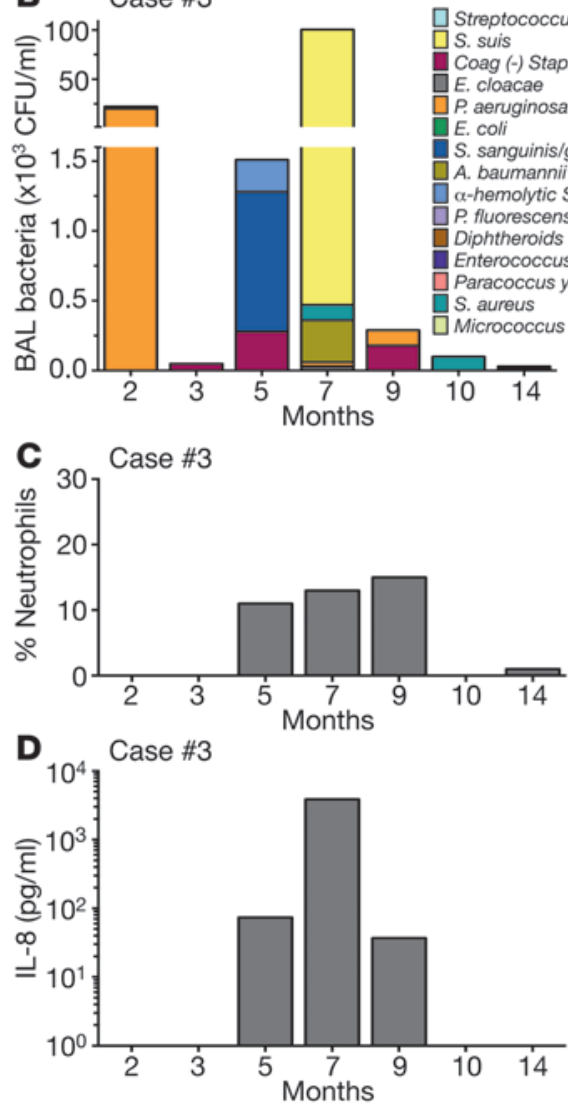
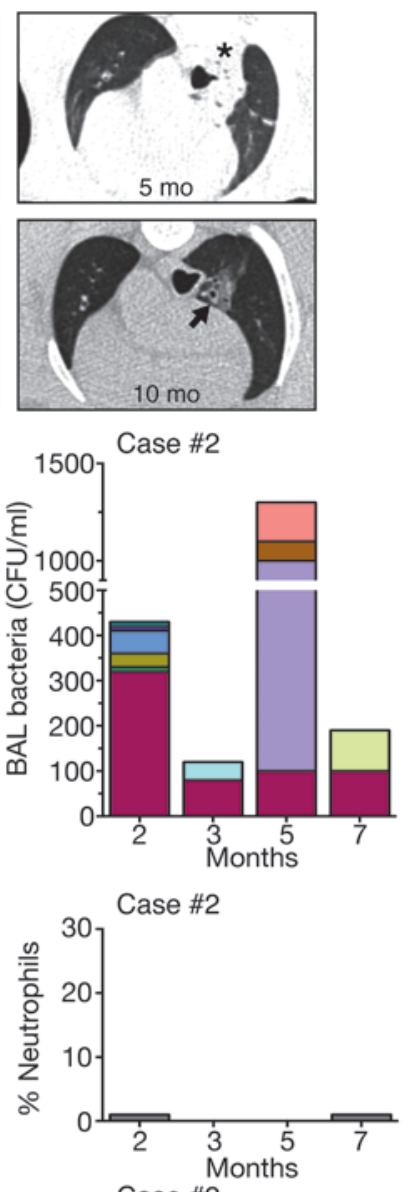

Case \#2

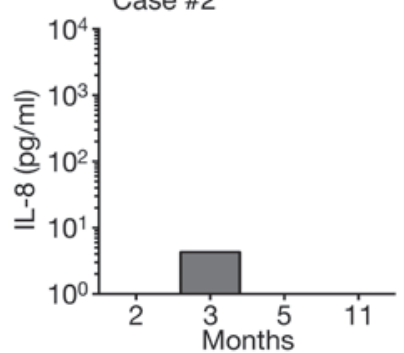

\section{Figure 6}

Airway disease is dynamic in $\mathrm{CFTR}^{-1-}$; $T g F A B P>p C F T R$ pigs. (A) Serial chest $x$-ray CT images from a CFTR ${ }^{-/-} ; T g F A B P>p C F T R$ pig (case no. 3). Airway wall thickening (arrows) was present. At 3 months, obstruction of the tracheal bronchus was observed, with collapse of the associated lung segment (asterisks). This persisted until 9 months and was resolving by 10 months. Airway wall thickening and parenchymal changes persisted. (B-D) Serial tracheal lobe BAL samples from 2 CFTR $^{-/-}$; $T g F A B P>p C F T R$ pigs (case no. 3 , left panels) (case no. 2, right panels). (B) Total number and different bacterial species recovered in the BAL liquid. Data are color coded to indicate individual species of bacteria. Percentage of neutrophils (C) and IL-8 levels (D) were determined on recovered liquid from the tracheal lobe BAL.
Histopathologic examination of the liver from older pigs revealed heterogeneous changes ranging from focal biliary cirrhosis to bridging changes indicative of multilobular cirrhosis (Supplemental Figure 3A). The pancreas showed evidence of progressive exocrine tissue loss and replacement by adipose tissue with prominent dilated ducts and acini (Figure 5B, Supplemental Figure 3B, and Supplemental Figure 4A), similar to that found in older CFTR ${ }^{-/}$ and $C F T R^{\triangle F 508 / \triangle F 508}$ pigs $(15,20)$. The gallbladders were small and variably filled with mucopurulent and necrotic cellular debris or mucus and inflammatory cells (Supplemental Figure 3C). We also observed variable changes along the length of the vas deferens; in some areas, the lumen was collapsed and filled with eosinophilic material that was PAS-positive, but more commonly the lumen was completely absent (Supplemental Figure 3D).

CFTR ${ }^{-1-} ; T_{g} F A B P>p C F T R$ pigs develop airway disease similar to that in bumans with CF. At necropsy, ethmoid sinuses were a common site of obstructive disease and frequently contained thick, tenacious mucocellular exudate (Figure 5C and Supplemental Figure 5A). Histopathological examination showed neutrophils and abundant mucus obstructing the air space (Figure 5D, Supplemental Figure 5, B and D, and Supplemental Figure 4B). Within the core of mucocellular debris, mixed populations of Gram-positive and Gram-negative bacterial colonies were often detected, as well as rare fungal elements (Supplemental Figure 5, E and F).

The tracheas were irregularly shaped, defects in the cartilage rings were present, and airway smooth muscle bundles appeared enlarged and prominent (Supplemental Figure 6). These findings are similar to those we previously reported for CF pigs (29). The lungs were affected in a heterogeneous pattern with mucus accumulation often centered on small bronchi to bronchioles. The abnormal airway mucus lesions ranged from mild lining of airways to completely obstructive changes, with mucocellular obstructions and concurrent atelectasis of lung tissues (Figure 5, E and F, Supplemental Figure 7, A-F, and Supplemental Figure 4C). 
While wispy mucus was occasionally detected lining larger airways in non-CF pigs, the nature and extent of the luminal mucus were distinct in CF pigs. The airway mucus of CF pigs frequently had lamellar to stringy appearance that sometimes appeared in small airways such as bronchioles that lacked overt mucinous change of the lining epithelium (Supplemental Figure 7F). In larger airways, the mucus lined the surface epithelium, which had multifocal goblet cell hyperplasia and mucus filling of submucosal gland ducts (Figure 5F and Supplemental Figure 7, A-D).

To follow the course of lung disease, we performed serial chest $\mathrm{x}$-ray CT imaging and bronchoalveolar lavage (BAL) in 2 longerlived $C F T R^{-1-} ; T_{g F A B P}>p C F T R$ pigs. Lung disease tended to be radiographically most evident in the tracheal lobe (Figure 6A). Evidence of persistent airway wall thickening was detected at 2 months of age. Radiographic changes were variably present over time, including patchy infiltrates and collapse of a tracheal lobe subsegment (Figure 6A). These findings are consistent with findings observed in human CF lungs (33).

Culture of BAL liquid showed variable numbers and species of bacteria over time (Figure 6B and Supplemental Figure 8). Consistent with our earlier studies of CF pigs (20), in case no. 2, we did not detect substantial BAL changes. Of note, at 3 different time points, BAL liquid from case no. 3 contained Pseudomonas aeruginosa (Figure 6B), an organism often isolated from human $\mathrm{CF}$ lungs $(34,35)$ and that we have previously isolated from porcine CFTR $^{-1}$ sinus (36). The absolute numbers of $P$. aeruginosa changed over time, and not all subsequent samples were positive. In case no. 3 , the waxing and waning numbers of BAL bacteria and chest CT abnormalities were paralleled by an increase and decrease in the percentage of neutrophils and IL-8 levels in BAL liquid (Figure 6, $\mathrm{C}$ and D). Interestingly, these changes occurred spontaneously despite lack of antibiotic use.

\section{Discussion}

CFTR expression in the intestine is sufficient to rescue meconium ileus in CF pigs. Finding that transgenic expression of CFTR induced anion transport in the newborn ileum and rescued the meconium ileus phenotype suggests that correcting $\mathrm{Cl}^{-}$and/or $\mathrm{HCO}_{3}{ }^{-}$transport across intestinal epithelia is sufficient to prevent meconium ileus. Moreover, intestinal CFTR expression prevented meconium ileus despite the presence of pancreatic disease. Thus, although there is a strong association between the occurrence of meconium ileus and pancreatic insufficiency $(12,37)$, that link is likely related to the presence of more severe CFTR mutations rather than to a causal role for pancreatic exocrine insufficiency in meconium ileus.

It is interesting to compare CF intestinal disease in humans, pigs, mice, and ferrets and the effect of an $i F A B P-p C F T R$ transgene. In humans, up to $20 \%$ of newborns have meconium ileus, and later in life, some people with CF develop distal intestinal obstruction syndrome (DIOS), an acute complete or incomplete fecal obstruction in the ileocecal region (38). It has been estimated to occur with an incidence of 6.2 episodes per 1000 patient-years and is usually treated conservatively. In CF pigs, intestinal disease resembles meconium ileus in humans with development in utero, occasional intestinal perforation prior to birth, and atretic bowel distal to the obstruction (7, 14-16). In addition, after the meconium ileus has been surgically bypassed, CF pigs may also develop a DIOS-like syndrome (14). Approximately $75 \%$ of CF ferrets are also reported to develop meconium ileus (39). In contrast, most CF mouse models have an intestinal phenotype resembling severe DIOS (40). For example, most mortality in CF mice occurs at the time of weaning, and only a small fraction die from intestinal disease shortly after birth. Transgenic expression of CFTR repairs the phenotypes in mice $(21,28,31)$ and pigs, and in a preliminary report, in ferrets (39). These results suggest that both meconium ileus and DIOS are caused by loss of intestinal CFTR anion transport. However, the data do not indicate that the pathogenic mechanisms are the same for the 2 manifestations. Understanding the mechanisms of meconium ileus will require studies at the time when it is developing in the fetus.

CFTR ${ }^{-1} ; \operatorname{TgFABP}>p C F T R$ pigs develop lung disease similar to that in buman $C F$. Although tissue-specific promoters can drive transgene expression at unintended tissue sites, our electrophysiological and histopathological studies indicate that the airways of the transgenic CF pigs developed disease like that seen in nontransgenic CF pigs $(15,20,36)$.

We previously reported that $\mathrm{CF}$ airway disease can vary over time $(15,20)$, and one of the $C F T R^{-1-} ; T_{g} F A B P>p C F T R$ pigs (case no. 3 ) provided a striking example of spontaneous variation in the severity of airway disease. Although the use of antibiotics and limitations of assays prevent such tracking in humans, these findings may have a counterpart in human CF. People with CF can manifest long periods of time with minimal or absent pulmonary symptoms, and there can be extended periods between exacerbations of pulmonary disease (41-43). These time-dependent variations suggest that not all host defense is disrupted by loss of CFTR. For example, we recently found that lack of CFTR-mediated $\mathrm{HCO}_{3}{ }^{-}$ transport and a decreased $\mathrm{pH}$ reduced, but did not abolish, bacterial killing by airway surface liquid antibacterials in CF pigs (44). The variation in bacterial species cultured from the CF lungs is also consistent with our earlier observations that the CF host defense defect does not favor a specific bacterial species (20).

There is a relationship between CFTR expression and the meconium ileus phenotype. With the development of CFTR-targeted therapies using either genetic or small molecule approaches, an important but unanswered question is how much CFTR is required to either alleviate or cure the disease. We recently showed that $C F T R^{\triangle F 508 / \triangle F 508}$ pigs develop lung disease like humans with CF, despite an apical membrane $\mathrm{Cl}^{-}$conductance approximately $6 \%$ of wild-type values (15). Those data suggest that more than $6 \%$ of wild-type CFTR channel function is required to prevent lung disease. Our current data indicate that approximately $10 \%$ of wild-type CFTR transcripts had a minor effect, but was insufficient to correct the meconium ileus phenotype. These values from airway and intestine are in reasonable agreement. We also found that approximately $20 \%$ of wildtype CFTR mRNA largely corrected the meconium ileus.

The approximately $10 \%$ and $20 \%$ of wild-type CFTR transcript levels translate to cAMP-stimulated short-circuit current of approximately $25 \%$ and $60 \%$, respectively. Note, however, that the short-circuit current measurements depend on basolateral membrane transport processes and other mechanisms in addition to CFTR. Thus, those values cannot be taken to mean that the epithelium had approximately $25 \%$ and $60 \%$ of CFTR function.

Comparisons between CFTR expression and correction of the meconium ileus phenotype have advantages and caveats. The advantages are as follows: (a) the site of CFTR expression in the crypts matches that of endogenous CFTR; (b) in contrast with previous studies in cultured airway epithelia in which a variable fraction of cells had wild-type CFTR expression (45) or CFTR overexpression (46), in this study, the population of cells expressed a variable fraction of wild-type CFTR; (c) we compared the percent- 
age of wild-type CFTR transcripts to a clinical phenotype rather than solely to an electrophysiological assay; (d) examining this relationship in CFTR-null pigs has the advantage that potential variations in processing of CFTR- $\Delta$ F508 do not introduce a confounding variable. Caveats include the following: (a) we focused on CFTR function in the ileum since the meconium ileus obstruction most commonly occurs in that intestinal segment (47); however, because the in utero pathogenesis of meconium ileus is unknown, it remains possible that CFTR function in other portions of the intestine might be more important for meconium ileus; (b) our estimates are derived from $\mathrm{CFTR}^{-/-}$pigs and not humans with $\mathrm{CF}$; although the porcine meconium ileus phenotype is very similar to that observed in humans, its penetrance is much greater than in humans (100\% vs. $15 \%-20 \%)(14-16,19)$; thus, intestinal anatomy, physiology, or genetic modifiers might differ in pigs and humans and thereby alter the relationship between CFTR function and meconium ileus severity; (c) our studies were conducted on newborn tissue, and we cannot say how much CFTR function is required during intestinal development.

The question about the amount of CFTR required to prevent intestinal disease was also tested by Dorin and colleagues (48). They crossed mouse models with different CFTR alleles that retained some CFTR expression. They suggested that approximately $5 \%$ of wild-type CFTR expression and approximately $20 \%$ of CFTR Isc was sufficient to almost completely rescue survival from intestinal disease. Those values are lower than our findings, perhaps because of differences in phenotype, meconium ileus versus DIOS, and/or perhaps because of other species differences.

Our results indicate that expressing CFTR in the intestine rescued the meconium ileus phenotype. Moreover, the $\mathrm{CFTR}^{-1}$; $T g F A B P>p C F T R$ pigs had pancreatic, liver, gallbladder, vas deferens, sinus, and lung disease comparable to that previously reported for $C_{F T R^{-/}}$and $C F T R^{\triangle F 508 / \triangle F 508}$ pigs $(15-18,21,30,36)$. This method offers a practical alternative to surgery for selectively correcting meconium ileus and thus may produce animals useful in understanding CF pathophysiology and testing new preventions and therapies.

\section{Methods}

CFTR ${ }^{-1-}$ TgFABP $>$ pCFTR vector construction. For generation of $\mathrm{CFTR}^{-1-\text {; }}$ $T g F A B P>p C F T R$ pigs, we transfected $C F T R^{-1}$ fetal fibroblasts with a transgenic construct containing the rat $i F A B P$ promoter (a gift from Jeffrey Gordon, Washington University, St. Louis, Missouri, USA) and porcine CFTR. Using the $\mathrm{PCI}$ vector (Promega), we first substituted the CMV promoter with the nucleotide sequence -1788 to +28 of the rat $i F A B P$ promoter. The porcine CFTR sequence was amplified from $p c D N A 3.1$-Kozak- $p C F T R$ plasmid using NheI and Xho1 and inserted into the redesigned $p C I-i F A B P$ vector with the same enzymes. Plasmid $p c D N A 3.1(+) H y g r o$ (Invitrogen) was digested with $N r u I$ and $E c o R V$ to remove the $C M V$ promoter ( $p c D N A 3.1\left(^{+}\right)$ Hygro $\triangle C M V$ ). The $i F A B P$-CFTR fragment was amplified from $p C I-i F A B P$ $p C F T R$ with PfuUltra Polymerase (Stratagene) and ligated with $p c D N A 3.1\left(^{+}\right)$ Hygro $\triangle C M V$. Restriction and sequence analysis were performed on the redesigned plasmid prior to use for transfection of fetal fibroblasts.

Fetal fibroblasts. We previously reported generation of $\mathrm{CFTR}^{-/-}$pigs (14). Fetal fibroblasts were isolated from day $35 \mathrm{CFTR}^{-/}$fetuses as previously described $(13,48)$. Cells were grown in F10 medium (Invitrogen) containing 20\% FCS and penicillin/streptomycin.

Transfection, selection, and Southern blot screening of clones. Fetal CFTR-1fibroblasts were electroporated with $15 \mu \mathrm{g}$ of linearized DNA from the $i F A B P-p C F T R$ transgenic construct and then cultured. After 48 hours, the medium was changed and supplemented with $100 \mu \mathrm{g} / \mathrm{ml}$ Hygromycin
(Invitrogen) for 14 days to select for antibiotic-resistant clones. Southern blot screening was performed on Hygromycin-resistant cells as previously described (13). Briefly, amplified whole genomic DNA was prepared from each clone and $20 \mu \mathrm{g}$ was digested with BanII overnight. Genomic digests were electrophoresed on a $0.8 \%$ agarose gel and transferred to a positively charged nylon membrane (Roche) using an alkaline transfer procedure. Blots were prehybridized for 30 minutes at $65^{\circ} \mathrm{C}$ in Rapid-hyb buffer (Amersham). The blot was then hybridized in Rapid-hyb buffer with a ${ }^{32} \mathrm{P}$-labeled probe specific for the entire exon 13 of porcine CFTR $\left(65^{\circ} \mathrm{C}\right.$, overnight). Two bands were anticipated on the blots: one at $2198 \mathrm{bp}$ for endogenous CFTR and the other at $3542 \mathrm{bp}$ for the transgenic CFTR.

Production of transgenic animals. Cryopreserved clones were thawed and resuspended in micromanipulation medium. Oocyte maturation, somatic cell nuclear transfer, surrogate preparation, and embryo transfer were all performed as previously described (13, 49-53).

Care of pigs. Piglets were delivered either via cesarean section on days 116-118 or naturally. After delivery, the piglets received colostrum. Some piglets received polyethylene glycol 3350 (Perrigo) orally. Shortly after birth, most piglets underwent a Gastrografin enema. This was repeated every 8-12 hours until all meconium was evacuated or the enemas failed to induce a response. Piglets were transitioned to milk replacer until mature enough to be placed on standard pig diets. In some litters, piglets received daily antibiotics for the first 7-14 days of life. All animals received oral pancreatic enzyme replacement therapy with meals and an oral proton pump inhibitor once a day.

BAL liquid collection and analysis. For BAL in the older pigs, animals were anesthetized with ketamine (15-20 mg/ kg, i.m.), xylazine (1-1.5 mg/kg, i.m.), and intravenous propofol. A flexible fiber optic bronchoscope (Pentax FB-10X) was inserted orally and passed through the vocal cords. The suction channel was not used until the tip of the bronchoscope was past the vocal cords. The bronchoscope tip was gently inserted and wedged into the tracheal bronchus airway for all animals. Three aliquots ( $10 \mathrm{ml}$ each) of sterile saline were instilled into the airway and lavage liquid was recovered with intermittent suction. The BAL liquid was pooled and immediately placed on ice, transported to the laboratory for processing (cell counts and microbiology studies), and stored at $-80^{\circ} \mathrm{C}$ for subsequent analysis.

The total number of recovered cells in BAL liquid was quantified with a hemacytometer, and morphologic differentiation of cells was performed on cytospin preparations that were stained with Diff-Quik Stain Kit (Baxter). Microbiologic studies were performed on collected BAL liquid. IL-8 levels were determined on recovered supernatant after centrifugation $(1600 \mathrm{~g}$ for 10 minutes) using a standard sandwich ELISA (R\&D Systems).

CT scanning. All sinus and chest $\mathrm{x}$-ray $\mathrm{CT}$ imaging was performed on sedated, spontaneously breathing animals using a 64-slice high-resolution CT (HRCT) scanner (SOMATOM 64; Siemens).

Production of primary cultures of differentiated airway epithelia. Epithelial cells were isolated from tracheas and nasal turbinates by enzymatic digestion, seeded onto permeable filter supports, and grown at the air-liquid interface as previously described (54). Differentiated epithelia were used at least 14 days after seeding.

Histopathologic analysis. At necropsy, pigs were examined for gross lesions, and the findings were documented. Tissues were collected and fixed in 10\% neutral buffered formalin usually for 48-96 hours. Tissues were then routinely processed, embedded, sectioned $(\sim 4 \mu \mathrm{m})$, and stained with routine histochemical stains such as H\&E for general examination. Special stains were selected for specific tissues/lesions and included amylase-pretreated PAS (dPAS), Gomori methenamine silver (GMS) stain, modified Gram stain, and Masson trichrome.

Meconium ileus scoring system. At necropsy, meconium ileus severity was scored by an investigator with substantial expertise in the gastrointestinal phenotype of newborn CF pigs. The following scoring system was 
employed: 3+, no correction of meconium ileus phenotype (newborn piglet failed to pass meconium spontaneously; intestinal diverticuli, intestinal atresia, and microcolon were present); $2+$, mild correction of meconium ileus phenotype (newborn piglet failed to pass significant meconium spontaneously; less severe intestinal diverticuli, intestinal atresia, and microcolon compared with $3+$ group); $1+$, moderate correction of meconium ileus phenotype (some newborn piglets passed meconium spontaneously; less severe intestinal diverticuli, intestinal atresia, and microcolon compared with $2+$ group but not completely normal as compared with wild-type pigs); normal: no meconium ileus phenotype (all newborn piglets pass meconium spontaneously; no intestinal diverticuli, intestinal atresia, or microcolon).

Microbiologic studies. Standard microbiologic techniques were utilized to identify and quantify bacteria present in BAL liquid samples. Samples were serially diluted and plated onto blood agar (tryptic soy agar with sheep blood; Remel), Colombia colistin-nalidixic acid agar (Remel), chocolate agar (Remel), mannitol salt agar (Remel), MacConkey agar (Remel), and Burkbolderia cepacia selective agar (Remel). Organisms were identified with standard microbiological procedures. Some identifications were confirmed by API 20E or API 20NE (bioMérieux), Vitek (bioMérieux), or 16S rRNA gene sequencing (University of Iowa Clinical Microbiology Laboratory and Iowa State University Diagnostic Laboratory).

Quantitative RT-PCR. Quantitative RT-PCR used TaqMan chemistry and an ABI 7500 Fast Real-Time PCR System to measure porcine CFTR mRNA. Briefly, tissue was collected in RNAlater (Ambion), and total RNA was isolated. First-strand cDNA was synthesized with random hexamers (SuperScript III). Sequence-specific primers and probes for porcine CFTR and $\beta$-actin were from ABI. For measuring CFTR mRNA, primer/probe sets annealing to exon 10 of CFTR (Ss03389420_m1, pCFTR) and $\beta$-actin (Ss03376160_u1, ACTB) were used in separate reactions. CFTR mRNA levels were normalized to $\beta$-actin. This normalized value for each tissue (ileum or trachea) was expressed relative to normalized $\mathrm{CFTR}^{++}$tissues to calculate the percentage of mRNA relative to wild-type levels (100\%).

Immunocytochemistry. Intestinal tissues were excised from newborn piglets, fixed 1 hour at room temperature in 2\% paraformaldehyde, and then kept overnight in ice-cold $30 \%$ sucrose before quick-freezing in OCT in an ethanol/ dry ice bath. Tissue segments were kept at $-80^{\circ} \mathrm{C}$. Tissues were cryosectioned into $7-\mu \mathrm{m}$ sections, permeabilized in $0.2 \%$ Triton X-100 (Thermo Fisher) in PBS, and blocked in Super-Block (Thermo Fisher) with $5 \%$ normal goat serum (Jackson ImmunoResearch Laboratories). Tissue sections were incubated for 2 hours at $37^{\circ} \mathrm{C}$ in anti-CFTR antibody 596 (CF Folding Consortium) and polyclonal antibody to $\beta$-catenin (Invitrogen; Zymed) (all at 1:100 dilution), followed by secondary antibodies (goat anti-mouse Alexa Fluor 488 and goat anti-rabbit Alexa Fluor 568 (Molecular Probes;Invitrogen) (1:1000 dilution). Sections were mounted with Vectashield containing DAPI (Vector Laboratories) to visualize nuclei. Images were acquired on an Olympus Fluoview FV1000 confocal microscope with a UPLSAPO $\times 60$ oil lens. Images were scanned sequentially at $2 \mathrm{~ms} /$ pixel. Figure $2 \mathrm{C}$ represents acquired $Z$-series.

Electrophysiological measurements of freshly excised tissues and cultured airway epithelia. Epithelial tissues were excised from the nasal turbinate, trachea, and ileum immediately after animals were euthanized. Tissues and cultured epithelia were studied in modified Ussing chambers (EasyMount Ussing Chamber System; Physiologic Instruments). For excised tissues, a chamber with a 2-mm diameter aperture was used. Transepithelial voltage was maintained at $0 \mathrm{mV}$ to measure short-circuit current (Isc) (VCC MC-8; Physiologic Instruments).
Trachea and cultured airway epithelia were bathed on both surfaces with solution containing the following: $135 \mathrm{mM} \mathrm{NaCl}, 2.4 \mathrm{mM} \mathrm{K}_{2} \mathrm{HPO}_{4}, 0.6 \mathrm{mM}$ $\mathrm{KH}_{2} \mathrm{PO}_{4}, 1.2 \mathrm{mM} \mathrm{CaCl}_{2}, 1.2 \mathrm{mM} \mathrm{MgCl}, 10 \mathrm{mM}$ dextrose, $5 \mathrm{mM}$ HEPES, $\mathrm{pH} 7.4(\mathrm{NaOH})$, at $37^{\circ} \mathrm{C}$ and gassed with compressed air. A standard protocol was the following: (a) measurements under basal conditions; (b) apical $100 \mu \mathrm{M}$ amiloride to inhibit ENaC Na+ channels; (c) apical $100 \mu \mathrm{M}$ DIDS to inhibit most anion channels other than CFTR; (d) apical $10 \mu \mathrm{M}$ forskolin and $100 \mu \mathrm{M}$ IBMX to increase cellular levels of cAMP leading to phosphorylation and activation of CFTR; (e) apical $100 \mu \mathrm{M}$ GlyH-101 (a gift from the Cystic Fibrosis Foundation Therapeutics and Robert Bridges, Rosalind Franklin University of Medicine and Science, North Chicago, Illinois, USA) to inhibit CFTR; and (f) basolateral $100 \mu \mathrm{M}$ bumetanide to inhibit $\mathrm{Na}-\mathrm{K}-2 \mathrm{Cl}$ transporter.

Ileum was bathed on both surfaces with a solution containing the following: $118.9 \mathrm{mM} \mathrm{NaCl}, 2.4 \mathrm{mM} \mathrm{K}_{2} \mathrm{HPO}_{4}, 0.6 \mathrm{mM} \mathrm{KH}_{2} \mathrm{PO}_{4}, 1.2 \mathrm{mM} \mathrm{CaCl}_{2}$, $1.2 \mathrm{mM} \mathrm{MgCl}_{2}$, and $25 \mathrm{mM} \mathrm{NaHCO}_{3}$ at $37^{\circ} \mathrm{C}$ and gassed with $5 \% \mathrm{CO}_{2} /$ air balance. A standard protocol was the following: (a) measurements under basal conditions; (b) apical $100 \mu \mathrm{M}$ amiloride; (c) apical $10 \mu \mathrm{M}$ forskolin and $100 \mu \mathrm{M}$ IBMX; (d) apical $100 \mu \mathrm{M}$ GlyH-101; (e) basolateral $100 \mu \mathrm{M}$ carbachol; (f) apical $100 \mu \mathrm{M}$ DIDS; (g) apical $5 \mathrm{mM}$ dextrose was added to assess tissue viability, and (h) apical $200 \mu \mathrm{M}$ phlorizin was added to block sodium/glucose transport.

Statistics. Data are presented for individual animals and as means \pm SEM. For comparisons between 2 or more groups, the Kruskal-Wallis ANOVA using Dunn's post-test or 1-way ANOVA using Tukey's post-test was used for nonparametric and parametric data, respectively. Differences were considered statistically significant at $P<0.05$.

Study approval. The University of Iowa and University of Missouri Animal Care and Use Committees approved all animal studies.

\section{Acknowledgments}

We thank Elizabeth Allard, Jennifer Bartlett, Martha Bennett, Cassie Dohrn, Lonnie Dowell, Michelle Griffin, Pamela Hughes, Theresa Mayhew, Andrew Michalski, Clifton Murphy, Paul Naumann, Bethany Redel, August Rieke, Viral Shah, Jered Sieren, Lee Spate, Ping Tan, Jennifer Teson, Alex Tucker, Aliye Uc, Eric Walters, Yimo Wang, Kristin Whitworth, Terry Yin, Xia Zhang, and the University of Iowa Office of Animal Resources for excellent assistance and advice. This work was supported by the NIH (HL051670, HL091842, DK054759) and the Cystic Fibrosis Foundation. D.A. Stoltz is supported by the Gilead Sciences Research Scholars Program in Cystic Fibrosis. M.J. Welsh is an Investigator of the Howard Hughes Medical Institute.

Received for publication January 22, 2013, and accepted in revised form March 7, 2013.

Address correspondence to: David A. Stoltz, Department of Internal Medicine, Carver College of Medicine, 440 EMRB, Iowa City, Iowa 52242, USA. Phone: 319.336.1620; Fax: 319.353.6406; E-mail: david-stoltz@uiowa.edu. Or to: Michael J. Welsh, Howard Hughes Medical Institute, Carver College of Medicine, 500 EMRB, Iowa City, Iowa 52242, USA. Phone: 319.335.7619; Fax: 319.335.7623; E-mail:michael-welsh@uiowa.edu.

\footnotetext{
1. Welsh MJ, Ramsey BW, Accurso F, Cutting GR. Cystic fibrosis. In: Scriver CR, Beaudet AL, Sly WS, Valle D, Childs B, Vogelstein B, eds. The Metabolic and Molecular Basis of Inherited Disease. 8th ed. New York, New York, USA: McGraw-Hill; 2001:5121-5189.

2. Rowe SM, Miller S, Sorscher EJ. Cystic fibrosis. NEngl J Med. 2005;352(19):1992-2001.
}

3. Quinton P. Physiological basis of cystic fibrosis: a historical perspective. Physiol Rev. 1999; 79(suppl 1):S3-S22.

4. van der Doef HP, Kokke FT, van der Ent CK, Houwen RH. Intestinal obstruction syndromes in cystic fibrosis: meconium ileus, distal intestinal obstruction syndrome, and constipation. Curr Gastroenterol
Rep. 2011;13(3):265-270.

5. Gelfond D, Borowitz D. Gastrointestinal complications of cystic fibrosis. Clin Gastroenterol Hepatol. 2013;11(4):333-342.

6. Rescorla FJ, Grosfeld JL. Contemporary management of meconium ileus. World J Surg. 1993; $17(3): 318-325$. 
7. Murshed R, Spitz L, Kiely E, Drake D. Meconium ileus: a ten-year review of thirty-six patients. EurJ Pediatr Surg. 1997;7(5):275-277.

8. Clarke LL, Harline MC. Dual role of CFTR in cAMP-stimulated $\mathrm{HCO} 3$ - secretion across murine duodenum. Am J Physiol Cell Physiol. 1998; 274(4 pt 1):G718-G726.

9. Clarke LL, Stien X, Walker NM. Intestinal bicarbonate secretion in cystic fibrosis mice. JOP. 2001; 2(4 suppl):263-267.

10. Harmon GS, et al. Pharmacological correction of a defect in PPAR-gamma signaling ameliorates disease severity in Cftr-deficient mice. Nat Med. 2010; 16(3):313-318.

11. Garcia MA, Yang N, Quinton PM. Normal mouse intestinal mucus release requires cystic fibrosis transmembrane regulator-dependent bicarbonate secretion. J Clin Invest. 2009;119(9):2613-2622.

12. Kristidis P, et al. Genetic determination of exocrine pancreatic function in cystic fibrosis. Am J Hum Genet. 1992;50(6):1178-1184.

13. Rogers CS, et al. Production of CFTR null and $\Delta \mathrm{F} 508$ heterozygous pigs by AAV-mediated gene targeting and somatic cell nuclear transfer. J Clin Invest. 2008;118(4):1571-1577.

14. Rogers CS, et al. Disruption of the CFTR gene produces a model of cystic fibrosis in newborn pigs. Science. 2008;321(5897):1837-1841.

15. Ostedgaard LS, et al. The $\Delta \mathrm{F} 508$ mutation causes CFTR misprocessing and cystic fibrosis-like disease in pigs. Sci Transl Med. 2011;3(74):74ra24.

16. Meyerholz DK, Stoltz DA, Pezzulo AA, Welsh MJ. Pathology of gastrointestinal organs in a porcine model of cystic fibrosis. Am J Pathol. 2010; 176(3):1377-1389.

17. Pierucci-Alves F, et al. Swine models of cystic fibrosis reveal male reproductive tract phenotype at birth. Biol Reprod. 2011;85(3):442-451.

18. Karimi A, Gorter RR, Sleeboom C, Kneepkens CM, Heij HA. Issues in the management of simple and complex meconium ileus. Pediatr Surg Int. 2011; 27(9):963-968.

19. Carlyle BE, Borowitz DS, Glick PL. A review of pathophysiology and management of fetuses and neonates with meconium ileus for the pediatric surgeon. J Pediatr Surg. 2012;47(4):772-781.

20. Stoltz DA, et al. Cystic fibrosis pigs develop lung disease and exhibit defective bacterial eradication at birth. Sci Transl Med. 2010;2(29):29ra31.

21. Zhou L, et al. Correction of lethal intestinal defect in a mouse model of cystic fibrosis by human CFTR. Science. 1994;266(5191):1705-1708.

22. Sweetser DA, Hauft SM, Hoppe PC, Birkenmeier EH, Gordon JI. Transgenic mice containing intestinal fatty acid-binding protein-human growth hormone fusion genes exhibit correct regional and cell-specific expression of the reporter gene in their small intestine. Proc Natl Acad Sci US A. 1988;85(24):9611-9615.
23. Gordon JI, et al. Tissue specific expression and developmental regulation of two genes coding for rat fatty acid binding proteins. J Biol Chem. 1985; 260(4):1995-1998.

24. Odes HS, et al. Cystic fibrosis transmembrane conductance regulator and $\mathrm{Na}+$ channel subunits mRNA transcripts, and Cl- efflux, show a different distribution in rat duodenum and colon. Acta Physiol Scand. 2003;178(3):231-240.

25. Strong TV, Boehm K, Collins FS. Localization of cystic fibrosis transmembrane conductance regulator mRNA in the human gastrointestinal tract by in situ hybridization. J Clin Invest. 1994;93(1):347-354.

26. Trezise AE, Buchwald M. In vivo cell-specific expression of the cystic fibrosis transmembrane conductance regulator. Nature. 1991;353(6343):434-437.

27. Levy E, et al. Localization, function and regulation of the two intestinal fatty acid-binding protein types. Histochem Cell Biol. 2009;132(3):351-367.

28. Ostedgaard LS, et al. Cystic fibrosis transmembrane conductance regulator with a shortened $\mathrm{R}$ domain rescues the intestinal phenotype of $\mathrm{CFTR}^{-/}$mice. Proc Natl Acad Sci U S A. 2011;108(7):2921-2926.

29. Meyerholz DK, et al. Loss of CFTR function produces abnormalities in tracheal development in neonatal pigs and young children. Am J Respir Crit Care Med. 2010;182(10):1251-1261.

30. Chen JH, et al. Loss of anion transport without increased sodium absorption characterizes newborn porcine cystic fibrosis airway epithelia. Cell. 2010;143(6):911-923.

31. Hodges CA, Grady BR, Mishra K, Cotton CU, Drumm ML. Cystic fibrosis growth retardation is not correlated with loss of Cftr in the intestinal epithelium. Am J Physiol Gastrointest Liver Physiol. 2011; 301(3):G528-G536.

32. Rogan MP, et al. Pigs and humans with cystic fibrosis have reduced insulin-like growth factor 1 (IGF1) levels at birth. Proc Natl Acad Sci U S A. 2010; 107(47):20571-20575.

33. Robinson TE. Imaging of the chest in cystic fibrosis. Clin Chest Med. 2007;28(2):405-421.

34. Burns JL, et al. Longitudinal assessment of Pseudomonas aeruginosa in young children with cystic fibrosis. J Infect Dis. 2001;183(3):444-452.

35. Rosenfeld $\mathrm{M}$, et al. Early pulmonary infection, inflammation, and clinical outcomes in infants with cystic fibrosis. Ped Pulmonol. 2001;32(5):356-366.

36. Chang EH, et al. Sinus hypoplasia precedes sinus infection in a porcine model of cystic fibrosis. Laryngoscope. 2012;122(9):1898-1905.

37. Blackman SM, et al. Relative contribution of genetic and nongenetic modifiers to intestinal obstruction in cystic fibrosis. Gastroenterology. 2006; 131(4):1030-1039.

38. Houwen RH, et al. Defining DIOS and constipation in cystic fibrosis with a multicentre study on the incidence, characteristics, and treatment of
DIOS. J Pediatr Gastroenterol Nutr. 2010;50(1):38-42.

39. Sun X, et al. Disease phenotype of a ferret CFTRknockout model of cystic fibrosis. J Clin Invest. 2010; 120(9):3149-3160.

40. Grubb BR, Gabriel SE. Intestinal physiology and pathology in gene-targeted mouse models of cystic fibrosis. Am J Physiol Cell Physiol. 1997; 273(2 pt 1):G258-G266.

41. Goss CH, Burns JL. Exacerbations in cystic fibrosis. 1: Epidemiology and pathogenesis. Thorax. 2007; 62(4):360-367.

42. Aaron SD, et al. Adult cystic fibrosis exacerbations and new strains of Pseudomonas aeruginosa. AmJ Respir Crit Care Med. 2004;169(7):811-815.

43. Rabin HR, et al. Pulmonary exacerbations in cystic fibrosis. Pediatr Pulmonol. 2004;37(5):400-406

44. Pezzulo AA, et al. Reduced airway surface $\mathrm{pH}$ impairs bacterial killing in the porcine cystic fibrosis lung. Nature. 2012;487(7405):109-113.

45. Farmen SL, et al. Gene transfer of CFTR to airway epithelia: low levels of expression are sufficient to correct $\mathrm{Cl}^{-}$transport and overexpression can generate basolateral CFTR. Am J Physiol Lung Cell Mol Physiol. 2005;289(6):L1123-L1130.

46. Zhang L, et al. CFTR delivery to $25 \%$ of surface epithelial cells restores normal rates of mucus transport to human cystic fibrosis airway epithelium. PLoS Biol. 2009;7(7):e1000155.

47. Mushtaq I, Wright VM, Drake DP, Mearns MB, Wood CB. Meconium ileus secondary to cystic fibrosis. The East London experience. Pediatr Surg Int. 1998;13(5-6):365-369.

48. Dorin JR, et al. A demonstration using mouse models that successful gene therapy for cystic fibrosis requires only partial gene correction. Gene Ther. 1996; 3(9):797-801.

49. Lai L, Prather RS. Production of cloned pigs by using somatic cells as donors. Cloning Stem Cells. 2003; 5(4):233-241.

50. Lai $L$, et al. Production of $\alpha$-1,3-galactosyltransferase knockout pigs by nuclear transfer cloning. Science. 2002;295(5557):1089-1092.

51. Lai L, et al. Generation of cloned transgenic pigs rich in omega-3 fatty acids. Nat Biotechnol. 2006; 24(4):435-436.

52. Machaty Z, Wang WH, Day BN, Prather RS. Complete activation of porcine oocytes induced by the sulfhydryl reagent, thimerosal. Biol Reprod. 1997; 57(5):1123-1127.

53. Im GS, et al. In vitro development of preimplantation porcine nuclear transfer embryos cultured in different media and gas atmospheres. Theriogenology. 2004;61(6):1125-1135.

54. Karp PH, et al. An in vitro model of differentiated human airway epithelia: methods and evaluation of primary cultures. In: Wise C, ed. Epithelial Cell Culture Protocols. Vol 188. Totowa, New Jersey, USA: Humana Press, Inc.; 2002:115-137. 